\title{
Por una nueva solidaridad: El Corno Emplumado y la conformación de una red de fraternidad intelectual (1962-1969)
}

\author{
For a New Solidarity: El Corno Emplumado \\ and the Formation of a Network of \\ Intellectual Brotherhood (1962-1969)
}

\author{
Grethel Domenech Hernández \\ (D) https://orcid.org/0000-0002-1259-9992 \\ Universidad Iberoamericana, México \\ domenech221b@gmail.com
}

Resumen: El presente trabajo busca analizar el proyecto de solidaridad intelectual que articuló la revista mexicana El Corno Emplumado (1962-1969), el cual formó parte de una amplia red intelectual de solidaridad que se estableció a nivel continental y con un carácter transnacional. En los estudios sobre la intelectualidad de los sesenta, las revistas de corte contracultural como El Corno Emplumado y los proyectos de solidaridad de tipo intelectual no han sido los tópicos más revisados, de ahí el alto valor de acercarnos a esta temática desde otros enfoques y actores. Como se verá en las siguientes páginas, el desarro-

* Maestra en Historia por la Universidad Iberoamericana. Estudiante del doctorado en Historia, Universidad Iberoamericana. Líneas de investigación: historia de los intelectuales e historia del tiempo presente.

cómo citar: Domenech Hernández, G. (2020) Por una nueva solidaridad: El Corno Emplumado y la conformación de una red de fraternidad intelectual (1962-1969). Secuencia (108), e1830. Dor: https://doi. org/10.18234/secuencia.v0i108.1830 
llo de un proyecto de solidaridad a través de una red intelectual representó una novedosa y diferente forma de establecer relaciones intelectuales para la época y produjo importantes momentos como el Movimiento de Nueva Solidaridad y el Primer Encuentro de Poetas.

Palabras clave: solidaridad; redes intelectuales; El Corno Emplumado.

Abstract: This paper seeks to analyze the intellectual solidarity project articulated by the Mexican magazine El Corno Emplumado (1962-1969), which formed part of a broad intellectual solidarity network established at the continental level, with a transnational nature. In studies on the intellectuality of the 1960s, countercultural magazines such as El Corno Emplumado and intellectual solidarity projects have not usually been the focus of inquiry, hence the value of addressing this issue from other approaches and actors. As will be seen in the following pages, the development of a solidarity project through an intellectual network represented a new, different way of establishing intellectual relationships for the time and produced important moments such as the New Solidarity Movement and the First Poets' Meeting.

Keywords: solidarity; intellectual networks; El Corno Emplumado.

Recibido: 29 de febrero de 2020 Aceptado: 20 de julio de 2020

Publicado: 30 de octubre de 2020

\section{PRESENTACIÓN}

T a mayoría de los autores ${ }^{1}$ que se han acercado al mundo intelectual latiLnoamericano de la década del sesenta coinciden en destacar la urgente revisión de conceptos y temas que asumieron los intelectuales de entonces y la fuerte emergencia de publicaciones que sirvieron de plataforma a debates culturales y políticos. La década de los sesenta fue la de la descolonización

${ }^{1}$ Por sólo mencionar algunos: Claudia Gilman, Rafael Rojas, Leonardo Candiano, Silvia Sigal, Beatriz Sarlo, Juan Carlos Quintero Herencia y Jorge Volpi. 
de África, la visita de Sartre a La Habana, el aumento de los debates sobre el tercer mundo, del movimiento hippie y de la liberación sexual. Los convulsos años, además de iniciarse con el triunfo de la revolución cubana, fueron testigos del alzamiento de guerrillas en Colombia, Bolivia, Uruguay y México, por sólo mencionar algunos ejemplos, y de una amplia ola de violencia política que incluyó golpes de Estados como los del Perú en 1962 y 1968, el de Argentina en 1966 o el de Brasil en 1964. El continente también se vio inserto en las dinámicas de la guerra fría, contienda ideológica y geopolítica que sin dudas fue más que una mera confrontación entre la URSS y Estados Unidos.

Este contexto de posibilidades creó las condiciones para que la solidaridad estuviera en el centro de los proyectos políticos, sociales y culturales del continente e incluso fuera de este. La revolución cubana activó también ciertos afectos, ilusiones, peregrinajes intelectuales, lecturas teóricas y posturas ideológicas que se esparcieron por todo el continente. Definiciones como solidaridad, intelectual, cultura política e izquierda comenzaron a cargarse de nuevos significados y posibilidades. El término solidaridad, asociado a la disposición y acción de ayudar a otros desinteresadamente o a ejercer la bondad y a los lazos sociales que unen a determinados grupos humanos, comenzó a tener un papel considerable en los discursos de la época. Con un marcado cariz político, su presencia en los discursos políticos e intelectuales fue cada vez más amplia.

Congresos, encuentros e instituciones asumieron la solidaridad como uno de sus ejes de discusión y acción. Los más conocidos fueron la Conferencia Tricontinental de los pueblos de África, Asia y América Latina, del 3 al 15 de enero de 1966, la cual daría paso a la creación de la Organización de Solidaridad de los Pueblos de África, Asia y América Latina (ospaAAL). A lo largo del continente se desarrollaron jornadas de solidaridad con Guatemala, el día de la solidaridad con el Congo o semanas de solidaridad en contra de la guerra de Vietnam. En todos se debatieron ideas como el imperialismo, el colonialismo, la guerrilla o los movimientos de liberación. La figura central que se tomó como paradigma sin duda alguna fue la de Ernesto "Che" Guevara. Por sólo citar un ejemplo al respecto, el mensaje del Che a la Tricontinental, una vez hecho público y distribuido a diversos países, fue reproducido en folletos y revistas por diferentes organizaciones que lo imprimieron en otros idiomas como el italiano, el árabe y el japonés. Como bien expresa Aldo Marchesi (2019), estos eventos fueron "fundamentales para la construcción de la noción de cercanía subjetiva en el mapa de la revolución latinoamericana” (p. 72). 
Pero, a pesar de ser esta solidaridad revolucionaria en torno a movimientos guerrilleros y de izquierda la más conocida y estudiada, hubo otros proyectos que la tomaron como eje desde enfoques más relacionados con el universo intelectual. Es importante por ello retomar la amplia circulación del término en los sesenta y las distintas acepciones que coexistieron en una misma época. Bien señala Kristina Pirker (2017-2018) que en cualquier análisis al respecto no se debe perder de vista lo que ella ha llamado dos planos: "[...] tanto la función del internacionalismo y de la solidaridad dentro de los mismos proyectos revolucionarios de Cuba y Centroamérica, respectivamente, como la recepción, apropiación e incluso resignificación de estos valores y representaciones por los y las activistas de la solidaridad transnacional inmersos en sus propios contextos políticos e historias nacionales" (p. 123).

En los últimos años, varias aproximaciones desde la Historia insisten en recuperar una mirada transnacional que reflexione en torno a la complejidad de procesos políticos y socioculturales que se esparcieron por todo el continente y así profundizar en conceptos y categorías (guerra fría, nueva izquierda, guerrilla, juventudes o solidaridad) que permitan un mayor alcance investigativo en torno a los fenómenos que se desplegaron y catalizaron tras 1959 en el continente. Más allá de la importancia de las organizaciones y encuentros de solidaridad mencionados anteriormente, me interesa aquí analizar el proyecto de solidaridad intelectual que articuló la revista mexicana El Corno Emplumado (1962-1969) el cual formó parte de una amplia red intelectual de solidaridad que se estableció a nivel continental y con un carácter transnacional.

Su idea de solidaridad de inmediato entró en consonancia con proyectos como el de la revista argentina Eco Contemporáneo y la mexicana Pájaro Cascabel, y durante sus primeros años contribuyó a conformar una importante red que dio lugar a sucesos como el movimiento Nueva Solidaridad o el Primer Encuentro de Poetas. Como se verán en las siguientes páginas, el desarrollo de un proyecto de solidaridad a través de una red intelectual representó una novedosa y diferente forma de establecer relaciones intelectuales para la época.

Antes de pasar a conocer el proyecto de solidaridad de El Corno Emplumado es importante ubicarnos en el campo intelectual mexicano en el que esta publicación ve la luz. En el México de los años sesenta del pasado siglo xx la joven generación intelectual enfocaba su proyección en grupos culturales y revistas literarias. Algunas de las más importantes fueron "La cultura en México", suplemento del semanario Siempre!, la Revista Mexicana de Literatura; 
Pájaro Cascabel, y La Palabra y el Hombre, de la Universidad Veracruzana, que había comenzado a circular en 1957 bajo la dirección de Sergio Galindo. Todas ellas fueron eco de la renovación literaria e intelectual que vivía el continente.

Por otro lado, existían aquellas publicaciones que funcionaban como órganos de difusión de instituciones gubernamentales o académicas. En este grupo se encontraban Cuadernos de Bellas Artes y Revista de Bellas Artes (apoyadas por el Instituto Nacional de Bellas Artes y la Secretaría de Educación Pública), Diálogos (editada por El Colegio de México), y la Revista de la Universidad de México (publicada por la Universidad Nacional Autónoma de México) (Silva, 2017, p. 96). Además de El Espectador-revista política con miembros simpatizantes de la izquierda como Víctor Flores Olea, Enrique Gonzales Pedrero, Carlos Fuentes, Francisco López Cámara y Luis Villorocreada en 1959, y la revista Política, la cual duró de 1961 hasta 1967. Este listado demuestra la importante relación que se dio entre diferentes comunidades intelectuales y revistas.

El Corno Emplumado se insertó en este amplio campo y desde un inicio demostró su originalidad y presencia. La revista bilingüe creada por Margaret Randall y Sergio Mondragón en 1962 nació con una intención transnacional de establecer vínculos con poetas de todo el continente. Según Mondragón, la publicación surgió con la idea de dar a conocer la poesía de las dos grandes lenguas del continente. Difundió traducciones de poesía en portugués y fue la primera en dar a conocer la poesía beat en los países de habla hispana (Pereira, Albarrán, Rosado y Tornero, 2018).

En la Enciclopedia de la Literatura en México El Corno Emplumado es definida como una

[r]evista bilingüe en español y en inglés, desligada en sus inicios, de todo vínculo ideológico o político y que a menudo habla de los problemas espirituales de nuestra época. La concepción y el material gráfico de la revista revelan la influencia de corrientes como el surrealismo. Según Sergio Mondragón, la publicación surgió con la idea central de dar a conocer la poesía de las dos grandes lenguas del continente. La revista difundió traducciones de poesía en portugués y fue la primera en dar a conocer la poesía beat en los países de habla hispana (Pereira et al., 2018).

Quienes conformaron El Corno Emplumado pertenecieron a una joven generación que comenzó a intervenir en el espacio público latinoamericano 
con inquietudes literarias y políticas. Margaret Randall (2015) emigró de Estados Unidos a tierras aztecas y trajo consigo sus propios referentes políticos: "También experimentaba un despertar de la conciencia política común a muchas personas de mi edad, jóvenes que sentían un inquieto inconformismo sofocado por el manto del periodo marcartista, aunque habíamos nacido demasiado tarde para ser víctimas directas del asedio represivo" (p. 100).

La posición de la revista en la dinámica intelectual mexicana como una publicación autónoma y con fuerte influencia de la Generación Beat ha llevado a considerarla una revista underground; no obstante, Randall (2015) nos menciona al respecto:

¿Era El Corno Emplumado una revista underground en la honorable tradición de tantas publicaciones de izquierda? No, porque se enviaba por correo, se vendía en librerías de muchas partes del mundo y las ideas políticas explícitas solo eran una parte de su contenido. La poesía era nuestro plato fuerte, y nuestro objetivo era llegar a la mayor cantidad posible de lectores. Aun así, como señalara Merton, tenía algo del espíritu underground si con eso nos referimos a la falta de ortodoxia, de reserva, de justificaciones. Lo que decidíamos imprimir a menudo procedía de underground literarios que se abrían espacio en todos los continentes (p. 108).

En la configuración discursiva de la revista, la poesía ocupó un lugar central, era el género por excelencia para plantear las inquietudes y compromisos de El Corno. La selección de poetas estableció una constelación de autores para los que la poesía fue, más que un medio de comunicación, una forma de vida. Cada número abría con una nota editorial que recogía el espíritu del ejemplar. También se publicaron ensayos, cuentos, entrevistas y reseñas. El Corno fue, en esencia, una revista de poesía, pero en sus notas editoriales, en la sección de correspondencia, y en algunos de los ensayos publicados podemos rastrear su idea de solidaridad intelectual tal como veremos más adelante.

La voluntad de El Corno de convertirse en un puente cultural e intelectual fue una de las principales motivaciones para realizar una revista bilingüe que tuvo como uno de sus objetivos fundamentales la traducción de textos "del norte hacia el sur y viceversa". Como bien diría Randall (2015), "Nos tomábamos muy en serio la idea de que éramos un puente entre culturas, ideologías, generaciones, usos del lenguaje y modos de construir un poema" (p. 111). El Corno fue una revista cosmopolita en muchos sentidos, no sólo 
porque Margaret Randall provenía de Estados Unidos, sino también a través de Mondragón y su gusto por las filosofías orientales. Se puede detectar en sus páginas un especial espíritu utópico en un sentido amplio. La referencia y elogio a culturas indígenas, al budismo, a la filosofía new age y un retorno a lo sagrado como prisma para entender la vida son muy propios de su discurso y la diferenció de otras revistas de los años sesenta en América Latina.

\section{REDES Y REVISTAS}

Al aproximarnos a relaciones transnacionales tanto intelectuales como de otro tipo es imprescindible el uso de la categoría de redes. Ella permite entender relaciones de tipo regional o global que se tejen mediante conexiones y se van estableciendo entre grupos o sujetos que confeccionan un entramado común en cuanto a discursos, metas e ideas. Como ya demostró el libro Redes intelectuales transnacionales en América Latina durante la entreguerra, compilado por Alexandra Pita (2016), pensar las redes en clave transnacional es fundamental. Tener en cuenta estas relaciones no significa un simple entendimiento de quién le escribía a quién, o quién se comunicaba con quién, sino analizar formas de relaciones culturales, personales o políticas que se establecen en determinadas condiciones.

El enfoque de redes también ha cobrado gran importancia en la historia de los intelectuales, pues permite acercarnos a ellos como sujetos que ejercen sus discursos y acción en una comunidad y en un espacio público. Los estudios apuntan la importancia que ha tenido para los intelectuales la conformación de redes pues, a través de ellas, logran establecer complejos espacios de organización, creación de revistas, encuentros, instituciones y difusión de publicaciones. ${ }^{2}$ Isabel de León Olivares (2017) señala que "la red intelectual funciona como una categoría dinámica, porosa y elástica que, entre otras cosas, podría reemplazar nociones como la de influencia y la de generación, dos pilares que durante mucho tiempo asistieron a la historiografía literaria latinoamericana" (p. 179).

${ }^{2}$ Al respecto, además de los trabajos de Alexandra Pita e Isabel de León, los libros Redes intelectuales en América Latina. Hacia la constitución de una comunidad intelectual, de Eduardo Devés-Valdés (2007) y Revistas en América Latina: proyectos literarios, políticos y culturales, coordinado por Regina Crespo (2010), resultan fundamentales para el estudio de redes intelectuales. 
En este entramado de redes y revistas, vale recalcar las propuestas de Alexandra Pita (2016), Regina Crespo (2010) y Fernanda Beigel (2003) y sus miradas a los estudios de publicaciones en América Latina. Por otro lado, la investigadora Claudia Gilman (2003) que, aunque no se encuentra propiamente en esta línea de redes intelectuales, es imprescindible para pensar desde una óptica transnacional términos como solidaridad, intelectual o compromisos. Su libro Entre la pluma y el fusil. Debates y dilemas del escritor latinoamericano es un punto de partida necesario a partir de su categoría de familia intelectual latinoamericana, la cual entra en estrecho diálogo con la de redes intelectuales.

Las revistas resultan fuentes privilegiadas para la reconstrucción de redes intelectuales, además de ser fuentes esenciales de la historia de los intelectuales, sus propias cualidades de medios y plataformas de expresión grupal y en la mayoría de las veces transnacional permiten el intercambio y la conformación de espacios que aglutinan voces y discursos. Funcionan "como nodos de redes, capaces de revelar los encuentros, intercambios, conexiones y relaciones entre las figuras del mundo intelectual que hicieron posible su existencia" (León, 2017, p. 184). Dichas publicaciones periódicas son releídas como espacios textuales de sociabilidad intelectual: lugares que, al posibilitar el encuentro de editores, publicistas, escritores, críticos, traductores y otros agentes culturales, se trasmutaron en puntos de reunión virtual, donde los miembros de un movimiento o una red podían darse cita "sin atender [a] las edades, lugares, posiciones sociales, y a veces ni jerarquías" (León, 2017, pp. 184-185). Asimismo, son complejos y diversos textos en constante revitalización por el público que las lee.

En este trabajo se van a entender como un universo conformado por las posturas de los escritores que en ellas se expresaron, teniendo en cuenta, fundamentalmente, el tipo de revistas que son. La publicación seleccionada no se inserta en la percepción común y actual de revistas intelectuales o académicas en las que se proponen textos de opinión y la redacción no se responsabiliza de estos. Es una revista pensada como un hogar de familia, más que como una plataforma de divulgación. Hay un vínculo generacional, ideológico, de intereses, de posiciones, literario y a veces hasta sentimental entre sus colaboradores. ${ }^{3}$ Como bien nos explica Inés de León,

${ }_{3}^{3}$ Margaret Randall y Sergio Mondragón, los fundadores de El Corno Emplumado, estuvieron casados durante casi toda la existencia de la revista. 
[p]ara autoras como Alexandra Pita, Regina Crespo, Susana Zanetti, las revistas constituyen "documentos de cultura" que permiten visualizar tanto los nombres de aquellos que participaban en una determinada red, como la dinámica de sus vinculaciones: las ideas, los discursos y las preocupaciones compartidas; las aspiraciones y los proyectos comunes; el mapa de lecturas que suministraban tópicos discursivos; sus lugares de enunciación y enlace; las posiciones y/o tensiones que guardaban tanto al interior como al exterior de los campos intelectuales a los que pertenecían o pretendían pertenecer (León, 2017, p. 185).

El nacimiento o la sustantivación del intelectual estuvo estrechamente ligado a la prensa y su desarrollo en la modernidad: prensa, vida moderna y ámbito de lo público eran fenómenos tan imbricados que resultaba imposible pensarlos por separado (Santos, 2002). Durante el siglo XIX la prensa se convirtió en una aliada esencial de la vida intelectual y asistió a su surgimiento, siendo su primer y principal instrumento de hacer valer su condición. Las revistas fueron además el principal medio de expresión de los grupos y actores intelectuales que cobraron fuerza en los sesenta latinoamericanos del siglo xx. Bien lo deja expresado Claudia Gilman (2003): "[...] analizar una revista de los sesenta/ setenta implica la necesidad de desplazarse por la gigantesca red de las revistas latinoamericanas del periodo. Seguramente, de allí derivan los límites del estudio de una revista en particular para determinar la lógica de constitución de campos de actores, que excede con mucho el límite de la revista" (pp. 22-23).

Fernanda Beigel (2003), en el ensayo Las revistas culturales como documentos de la historia latinoamericana, argumenta el papel de estas en la expresión pública intelectual latinoamericana al decir "nos conectan de modo ejemplar, no sólo con las principales discusiones del campo intelectual de una época, sino también con los modos de legitimación de nuevas prácticas políticas y culturales" (p. 7), lo cual las convierte en una fuente histórica significativa y necesaria a la hora de abordar los principales conflictos de los años a estudiar. En la década del sesenta las revistas se convirtieron en un instrumento de transformación y participación en la realidad. La importancia de estudiarlas para la historia de los intelectuales en ese momento radica justamente en que fueron el medio que utilizaron los intelectuales, conscientes o no, para ser intelectuales.

En este sentido, y relacionado con los vínculos entre revistas y redes, parto de una de las principales premisas del texto Redes intelectuales transnacionales en América Latina: 
[...] las publicaciones periódicas no fueron solo un soporte de red, es decir, un mero reflejo de su actividad, sino que -siguiendo la idea de Pierre Bourdieu para estudiar el concepto de habitus- son "estructura estructurada y estructurante". Esto implica entender que las revistas son el soporte material de una estructura en sí misma, que se genera a través de la estructuración implícita en las prácticas culturales, y que es factible analizar el espacio de una red intelectual como el aspecto estructurante que nace de esta interacción (Pita, 2016, p. 6).

Otra de las propuestas más significativas y desarrolladas para el estudio de redes ha sido enfocar "el análisis en los bienes simbólicos -textos, ideas, discursos, objetos, imágenes- que, precisamente, circulan y se distribuyen gracias al funcionamiento de las redes forjadas entre autores o entre campos intelectuales diversos y distantes" (León, 2017, p. 181).

Para el caso del estudio de la solidaridad intelectual que promovió y articuló El Corno valdría la pena preguntarse si es posible pensar la solidaridad como un bien simbólico de las redes intelectuales que estableció y, ¿en qué sentido puede ser la solidaridad en los años sesenta del pasado siglo un bien simbólico? La definición de bien simbólico viene, principalmente, de los trabajos del sociólogo John B. Thompson (2002) en especial su libro Ideología y cultura moderna. Teoría crítica social en la era de la comunicación de masas, donde nos dice:

En este sentido, los fenómenos culturales pueden considerarse como formas simbólicas en contextos estructurados; y el análisis cultural puede concebirse como el estudio de la constitución significativa y la contextualización social de las formas simbólicas. [...] La contextualización social de las formas simbólicas también implica que éstas pueden transformarse en objetos de complejos procesos de valoración, evaluación y conflicto (p. XXIV).

La idea de solidaridad adquirió una importante presencia en los discursos políticos, culturales e intelectuales a partir de los sesenta. La mayoría de los proyectos que se enfocaron en cambios sociales de corte de izquierda o que tomaron la revolución cubana como referente utilizaron a la solidaridad como una de sus principales formas de relacionarse y a la vez expandir la idea de la revolución.

Así, la solidaridad se convirtió en un bien simbólico de los sesenta que ensalzaba a sus portadores y los dotaba de pertenencia a un discurso transna- 
cional. En el caso de las revistas generó un estatus intelectual, hablar de solidaridad con movimientos revolucionarios, con guerrilleros, con las marchas de protesta en Estados Unidos y con la revolución cubana significaba formar parte de una lucha latinoamericana y poseer una conciencia histórica de la época. La solidaridad reafirmó discursos de corte contraculturales o incluso de izquierda, era una cualidad que los sectores reaccionarios no poseían a los ojos de este contexto; de ahí que manifestarla y articularla era una reafirmación de pertenencia. Además, fue un capital relacional que permitió entablar diálogos y formar redes con otros grupos o personas que manejaban ese mismo lenguaje. A partir de la solidaridad intelectual, El Corno se relacionó con los nadaístas en Colombia, participó en la organización del Primer Encuentro de Poetas o fomentó la lectura en distintas latitudes de los miembros de su red intelectual.

Hoy, que varios trabajos se aproximan al universo intelectual de la segunda mitad del siglo $\mathrm{xx}$ desde nuevas categorías: redes intelectuales, compromisos o familia intelectual latinoamericana, me gustaría añadir a esta lista la de solidaridad. Las anteriores y esta ayudan a comprender nuevas dimensiones de ese convulso mundo que se gestó en revistas, polémicas, encuentros y discursos a partir de 1959 en América Latina. Todas estas categorías están entrelazadas entre sí y el estudio de una lleva indudablemente a otra. En este trabajo, a pesar de centrarnos en la solidaridad intelectual, estableceremos conexiones con los otros nodos de la red intelectual que tejió El Corno, siendo ello de gran importancia para un mayor entendimiento de su proyecto de solidaridad.

A partir de la propuesta del estudio de redes me interesa analizar cómo El Corno Emplumado articula, teje y posibilita una red intelectual de solidaridad: leer la solidaridad en clave de red a través de El Corno Emplumado. Para ello primero hay que tener en cuenta qué ideas o preceptos tenía El Corno sobre la solidaridad, qué tipo de solidaridad va a articular y con qué objetivos, y, segundo, cómo se despliega esta a través de una red intelectual. El criterio de selección de esta revista como eje de análisis responde al papel destacado que tuvo en la conformación de una red intelectual entre distintos grupos y revistas del continente y su intensa reflexión y acción por un movimiento de solidaridad intelectual. 


\section{LA SOLIDARIDAD INTELECTUAL DE EL CORNO}

En la nota editorial con la que abría su primer número, El Corno Emplumado expresaba cuáles eran sus intenciones y el carril que deseaban que la revista tomara:

EL CORNO EMPLUMADO-THE PLUMEd CORN es una revista de poesía, prosa, cartas y arte del Continente [...] editada en español e inglés y publicada en la Ciudad de México [...] ésta es una revista cuyas páginas están dedicadas a servir a la palabra y con las cuales se pretende crear la publicación que hace falta... hoy día, cuando las relaciones entre los países de América son peores que nunca, esperamos que EL CORNO EMPLUMADo sea la mejor prueba (no política) de que todos somos hermanos [...] Deseamos que nuestra revista sea el principio de un plan para establecer una casa editorial y después, más tarde, MUCHO MÁs QUE ESO. ${ }^{4}$

En clara declaración de propósitos, típico de las primeras notas editoriales en una revista, El Corno establecía su carácter solidario, su intención de unidad intelectual y su compromiso con la palabra para transformar la vida. Estos contenidos fueron esenciales a lo largo de toda su existencia. Respecto a su trasfondo ideológico, El Corno mantenía sus puertas abiertas a toda presencia que reverenciara una libertad de la expresión, una actitud crítica y una búsqueda de la transformación de la vida y la sociedad. Uno de los mayores paradigmas que siguió El Corno fue la Generación Beat, sobre todo a partir de la presencia de Margaret Randall como una de sus directoras.

Desde un inicio podemos ver el llamado que hacían sus editores a estrechar vínculos entre los escritores de América, en todo su sentido continental. Una perspectiva transnacional que respondió a la propia idea de solidaridad y que es insoslayable de la connotación que cobró el propio término en la época. Para El Corno era fundamental ser punto de encuentro de nuevas voces, punto de hermandad de los poetas y punto de convergencia de la comunidad intelectual:

From a Catholic seminay in the jungles of Colombia, a Buddhist monestary in Kyoto, from a carpenter in Nicaragua, a student of physics in New Mexico, a

4 “Nota de los editores”, El Corno Emplumado, núm. 1, 1962, p. 5. 
poet prophet wandering through the Himalayas, Trappist monk in Kentucky, profesor of German in a college in upstate New York, bookseller in San Francisco, poets in London, Buenos Aires, Maine, Montevideo, Oslo, Cuba, New Jersey... comes the new poetry. ${ }^{5}$

Un concepto esencial para comprender la idea de solidaridad de El Corno es el de hombre nuevo. Este entra en constante diálogo con la visión de la revista sobre la labor del intelectual, el escritor o el hombre nuevo. En el proyecto intelectual de El Corno, la solidaridad, la revolución espiritual y el hombre nuevo eran indisolubles. Para sus colaboradores no se trataba solamente de un nuevo intelectual miliciano o un nuevo político de la sociedad revolucionaria, sino un hombre total que comprendía el cambio de paradigmas en el ser intelectual que la nueva época inspiraba:

Vivimos en una nueva era, la Era del Hombre. Es nueva porque así lo han determinado los procesos cósmicos, pero lo es también porque un hombre nuevo ha aparecido -y está apareciendo- en nosotros. Y los poetas, que son la voz de la tribu cantan a este hombre nuevo; o mejor: desde este hombre nuevo [...] EL CORNO EMPLUMADO es un instrumento para transmitir la nueva palabra, que es decir, el nuevo espíritu. ${ }^{6}$

En la tesis Texto, contexto e indices de El Corno Emplumado (1962-1969), de Gabriela Silva (2017), la autora nos expone que los primeros años de El Corno Emplumado están regidos por una idea central: el hombre contemporáneo es capaz de tomar consciencia plena de sí y de transformarse en un ser superior en un sentido espiritual. La encarnación de estos principios fue la noción del hombre nuevo que en El Corno Emplumado alcanzó una intención no sólo revolucionaria en un sentido de movimientos políticos, sino también una connotación espiritual (p. 145). La concepción del hombre nuevo fue muy popular en la década y sería retomada posteriormente por Ernesto "Che" Guevara en su trabajo "El socialismo y el hombre nuevo en Cuba".

Otra de las ideas centrales de El Corno y la cual entró en diálogo directo con la de solidaridad fue la de "época". Para la revista, la época (un espacio y un tiempo) no era sólo un lugar, sino también, la causa de un nuevo

5 "Nota de los editores", El Corno Emplumado, núm. 6, 1963, p. 6.

6 "Nota de los editores", El Corno Emplumado, núm. 6, 1963, p. 6. 
movimiento intelectual, una nueva etapa de vida. La frase "nuestro tiempo" común en los proyectos intelectuales desde mediados de siglo, ${ }^{7}$ hacía alusión a una gran responsabilidad intelectual y justo una de las responsabilidades principales que se adjudicó El Corno fue establecer esta hermandad o fraternidad entre escritores y artistas del continente. En El Corno la condición intelectual significaba una unión de fuerzas continentales, una solidaridad que aunara con la época que vivía el hombre latinoamericano:

Es necesario que la expresión de nuestro tiempo sea vista de la misma manera. Nuestra época Cuba, África, Chessman, la bomba atómica, protestas colectivas, expresionismo abstracto, música electrónica, un millón de niños nacidos diariamente-reduce nuestra acción a una locura que fractura la luz en que nos movemos. Las respuestas que buscamos se nos ocultan tras la maquinaria del dogma, los viejos odios y el funcionalismo de la sociedad. EL CORNO EMPLUMADO continuará sobre la base de que más allá de esas categorías estamos unidos por una fraternidad llamada arte. ${ }^{8}$

En su segundo número, la revista hacía un llamado directo a la solidaridad, el lenguaje utilizado resonaba en un horizonte de fraternidad y hermandad que emergía en los sesenta con especial fortaleza:

Nuestro órgano de difusión El CORNo EMPLUMADo necesita de la ayuda de todos nosotros hermanos de la hora presente en la que se escuchan voces tan contradictorias y en la que los vientos presagian tormentas y tropiezos. Las máscaras están cayendo con sorprendente rapidez, los acontecimientos angustian, estamos en una paradoja, la dolorosa condición del hombre se agudiza. Una cosa precisa el mundo: nuestra sinceridad y desprendimiento interior. Ello es la fraternidad. El Corno Emplumado esta así abierto a todas las voces y todas las congojas. ${ }^{9}$

7 Pensemos en Les Temps Modernes, primer gran paradigma de revistas comprometidas o en la revista cubana Nuestro Tiempo, creada en 1948 por varios de los que después del triunfo de la revolución serían colaboradores de Lunes de Revolución y Casa de las Américas.

8 "Nota de los editores", El Corno Emplumado, núm. 3, 1962, p. 5. Las cursivas son del texto original.

9 "Nota de los editores", El Corno Emplumado, núm. 2, 1962, p. 5. 
La condición solidaria y esta intención de cultivar una red intelectual convirtieron las páginas de El Corno en una plataforma de divulgación de otros movimientos o autores que partían de los mismos principios que la revista mexicana. Uno de los casos más significativos fue el de los nadaístas de Colombia. Con un discurso contracultural y una visión escéptica y a la vez vitalista, el nadaísmo se mostraba como un hermano literario de El Corno. En el número 7 la publicación se hacía eco del movimiento y publicaba un Manifiesto Nadaista que enarbolaba al hombre como centro de la revolución:

[...] todo lo que tenemos que ofrecerle a la juventud es la locura, pues es necesario enloquecernos antes de que llegue la guerra atómica [...] Y más allá del horizonte de la locura cuál es realmente el fin del nadaísmo? Y yo les diré: El Nadaísmo no tiene fin, porque es infinito. Nosotros nos contentamos con progresar devotamente hacia la locura y el suicidio. Hacemos el mal porque el bien no sienta a nuestro heroísmo. ${ }^{10}$

En su afán por establecer la solidaridad intelectual entre los escritores del continente, El Corno se convirtió en plataforma de redes, encuentros y divulgación de escritores y artistas alineados a estas mismas intenciones. Esta red se estableció tanto hacia el sur, como hacia el norte. El reclamo de solidaridad era un lenguaje común incluso en diferentes idiomas. En el número 9 publicaban la carta que desde Washington, D.C. les envío Alex Rode, subdirector de ouTCKY ${ }^{11}$ en la que decía:

Amigos de los países americanos: La revolución poética, la revolución del espíritu que todos los de nuestra generación ya sienten en lo que leen y en lo que escriben, ahora nos confronta con una decisión. Nos quedaremos segregados y relativamente ineficaces o podremos unirnos (sin rendir el poder de la voz solitaria) en un coro artístico de tanta fuerza que las barreras de los carniceros de la vida se caigan en todas partes del continente. ${ }^{12}$

${ }^{10}$ Gonzalo Arango, "Manifiesto Nadaísta”, El Corno Emplumado, núm. 7, 1963, p. 92.

${ }^{11}$ Outcry fue una de las revistas más activas del mundo contracultural estadunidense en los años sesenta.

${ }^{12}$ Alex Rode, “Carta”, El Corno Emplumado, núm. 9, 1964, p. 141. 
Para Rode, Margaret Randall y Sergio Mondragón en México eran el "contacto más importante para los escritores de ambas américas" [sic], de ahí el interés en trazar líneas de trabajo desde ambas latitudes en función de empoderar la red de poetas. Con este fin le proponía a El Corno las siguientes tareas:

1. Cambiar con Uds. copias de cada número de nuestras propias revistas. 2. Cambiar anuncios. 3. Recibir de su país manuscritos de los mejores escritores jóvenes para traducirlos al inglés y publicarlos en oUTCKY y en otras revistas de mismo tipo en Norteamérica. 4. Enviar a Uds. manuscritos en inglés de nuestros nuevos escritores, para que se traduzcan y publiquen en su país. Lo único que importa es que estamos unidos en estos principios: contra los poderes antivitales, antisexuales y antipoéticos; aliados a las fuerzas para la paz, la libertad y el individualismo. Diferencias estéticas no importan. Diferencias políticas son preocupaciones de la generación pasada. Deferencias nacionales ya no tienen ningún sentido. ${ }^{13}$

Además de la recepción y circulación de El Corno por Estados Unidos lo cual permitió tejer esta red intelectual de solidaridad a ambos hemisferios, hacia el sur se establecieron importantes lazos que dieron valiosos momentos de solidaridad en los sesenta. El mapa de esta red, que tuvo uno de sus principales anclajes en El Corno, contó también con Eco Contemporáneo desde Buenos Aires, El pez y la serpiente en Nicaragua, el grupo artístico literario El techo de la ballena (Edmundo Aray y Juan Calzadilla) en Venezuela, los Nadaístas en Colombia y Pájaro Cascabel, ${ }^{14}$ homóloga de El Corno en la Ciudad de México. A partir de ellas podemos trazar la cartografía transnacional en la que se movieron sus escritores y artistas que tuvo como centro de su discurso el arte y la literatura como formas de transformar la vida y llevar a cabo una revolu-

${ }^{13}$ Alex Rode, "Carta", El Corno Emplumado, núm. 9, 1964, pp. 149-150.

${ }^{14}$ Revista fundada en 1962 por Thelma Nava y el crítico literario Luis Mario Schneider. Junto con El Corno Emplumado, Cuadernos del Viento y El Rehilete, formó parte de las revistas poéticas de los sesenta que se alzaban como plataforma de una joven generación de escritores. En 1967 llegó a su último número, pues después del apoyo al movimiento estudiantil del 68 no le fue posible lanzar nuevos ejemplares. Entre sus principales colaboradores se encontraban Juan Bañuelos Jaime Sabines, Homero Aridjis, Ernesto Mejía Sánchez, Marco Antonio Montes de Oca, Efraín Huerta y Salvador Novo. 
ción espiritual y la solidaridad intelectual. Todos partían de "un despertar de conciencia”, ese era la gran clave que los unía.

De estas revistas, El Corno fue la más estructurada, mantuvo su publicación cuatrimestral durante sus años de existencia logrando así una regularidad y un espacio legítimo como publicación literaria. Además, como bien expresa Valeria Manzano (2017), "Aunque su impacto dentro del campo literario mexicano haya sido menos significativo que el del Nadaísmo y los balleneros en Colombia y Venezuela, El Corno Emplumado fue, de todas las iniciativas aquí analizadas, la que más aportó a la 'interamericanidad' de las variantes contraculturales" (p. 10).

Esta idea es central para entender por qué articular el análisis de esta red desde El Corno. De cierta forma la revista mexicana fue la que posibilitó el contacto con los otros puntos de anclaje tanto hacia el norte como hacia el sur.

Para delinear el itinerario de esta red debemos fijarnos atentamente en Miguel Grinberg, poeta argentino que en 1961 creó la revista Eco Contemporáneo junto con Antonio Dal Masetto, la cual, desde su primer número, se definía como una revista "interamericana". Este primer número incluía un ensayo sobre Poesía Brasileña, un texto sobre los nadaístas en Colombia, el poema America de Allen Ginsberg, selección de poesía de Perú y el texto vivencial de LeRoi Jones, 15 "Cuba 1960". Además de esto, Grinberg era un gran seguidor de la Generación Beat, lo cual lo emparentaría aún más con la revista mexicana. Grinberg sería un habitual de El Corno, tanto en las páginas centrales como en la sección de cartas. Su contacto con Thomas Merton y su viaje a Estados Unidos, para el cual contó con el apoyo de Margaret Randall, afianzaría más sus lazos. La red se iría tejiendo así cada vez más. Para El Corno, Miguel Grinberg o Ernesto Cardenal, representaban la mejor versión de la unidad entre los poetas y eran referenciados como actores centrales de esta solidaridad:

Y Miguel Grinberg, un poeta de Argentina, acaba de fundar la liga internacional de poetas (cuyo presidente ya aceptó ser Henry Miller), y Ernesto Cardenal en su monasterio de Colombia sigue diciendo que la verdadera Unión Panamericana es la de los Poetas, y nosotros le creemos, y al final de este Corno va

${ }^{15}$ Nombre de nacimiento del poeta afroamericano Amiri Baraka. Autor del poemario Preface to a Twenty-Volume Suicide Note (1961) y de los ensayos de crítica musical Blues People (1963) y Black Music. Visitó Cuba en julio de 1960 y escribió el ensayo "Cuba Libre" publicado en la revista Evergreen Review en diciembre de 1960. 
una lista de revistas que sin ponerse de acuerdo entre sí transmiten la luz y quieren la paz y creen en el hombre. ${ }^{16}$

Después de crear Eco Contemporáneo, Grinberg comenzó a gestar la Liga Internacional de Poetas, de la cual Henry Miller y Thomas Merton aceptaron ser presidentes honorarios. Referente a esa misma Liga, en una carta Miguel Grinberg les decía a Margaret y Sergio que la función y el objetivo de la Liga debería ser:

[...] algo que deberá fundamentar la real y legítima unión de las naciones, poetas hay en todo sitio, en cada resquicio del planeta hay cantos disgregados, a nosotros nos toca crear el nexo, restituirnos al sitio que nos corresponde, reinstaurar la fraternidad, detener la maquinaria de la guerra, anular al megatón, empiezo a despachar cartas a los poetas de América y Europa, te pido me contestes tu opinión sobre esto que te digo...! [sic]. ${ }^{17}$

Líneas después, Grinberg les decía que ya había comenzado a gestionar el primer congreso de la Liga y que podía ser en Río de Janeiro "donde no serían tildados de comunistas”.

\section{MOVIMIENTO DE NUEVA SOLIDARIDAD}

Las conversaciones para gestar un encuentro se concretaron y para 1962 se dio a conocer el movimiento Nueva Solidaridad, bajo la tutela de Grinberg pero al que tributaban El Corno, los nadaístas de Colombia y "El Techo de la Ballena” en Venezuela. La alianza de poetas continuó gestando momentos y trabajando en la fraternidad y hermandad defendida por El Corno, y después de labores organizativas por parte de Grinberg, El Corno, Thelma Nava y las demás revistas implicadas, en 1964 tuvo lugar el Primer Encuentro Americano de Poetas. ${ }^{18}$ El encuentro, a pesar de que se había pensado hacer en Brasil,

16 "Nota de los editores", El Corno Emplumado, núm. 7, 1963, p. 5.

${ }^{17}$ Miguel Grinberg, "Carta”, El Corno Emplumado, núm. 7, 1963, pp. 173-174.

${ }^{18}$ Antes del encuentro de los poetas en la Ciudad de México podemos rastrear otras iniciativas similares que, aunque gestadas fuera de esta red, nos remiten a un ambiente de época y a cómo están pensando los intelectuales sus formas de vincularse. En este sentido podemos mencionar el Primer Encuentro de Escritores Americanos, convocado en enero de 1960 por el 
finalmente tuvo lugar en la Ciudad de México. Este cambio respondió primero a cuestiones de logística y, segundo, a las facilidades que podían brindar las conexiones de El Corno Emplumado y Pájaro Cascabel en territorio mexicano.

La dinámica del suceso fue al estilo de sus participantes: poética, reflexiva y vital. Como bien recordaría El Corno: "Hubo allí de todo: micrófonos, reporteros, fotógrafos, saboteadores, gritos, malentendidos, y en más de una ocasión las sesiones se convirtieron en mítines políticos". ${ }^{19}$ Los espacios del encuentro fueron el Círculo de Periodistas Mexicanos y picnics en el Bosque de Chapultepec, participaron poetas de aproximadamente quince países de América Latina. En el número 10, de abril de 1964, El Corno resumía detalladamente lo sucedido en el encuentro:

Estas personas, misteriosamente conectadas entre sí en los últimos dos años por una red de cartas y revistas de poesías, vendieron sus libros y máquinas de escribir, aprovechando el plan de "vuelve ahora y pague después", y llegaron a esta ciudad durante la primer semana de febrero. Aquí durmieron en hoteles baratos, sobre petates o camas extras en las casas de los poetas mexicanos, y compartieron arroz y frijoles diariamente con nosotros. ${ }^{20}$

La nota redactada por El Corno sobre el encuentro nos habla de cómo entendían la transformación y el ser intelectual a través de una revolución interior:

Lo mejor de este encuentro no sucedió en las sesiones del Club de Periodistas. Sucedió en el bosque de Chapultepec, en las ruinas de Malinalco, en las calles y parques, en las casas de los poetas, en caminatas nocturnas. Fue allí donde se intercambiaron palabras e ideas. Donde se hicieron confesiones y preguntas. Donde comprobamos que estamos en el centro de una revolución espiritual que los poetas, por supuesto, son los primeros en redactar y descifrar. Fue así, casi de boca a oídos, como se dijo que el mundo exterior no es otra cosa que fiel reflejo de nuestra situación interna, que en nuestras manos

poeta chileno Gonzalo Rojas en la Universidad de Concepción y el Congreso de Intelectuales de Concepción en 1962, organizado también por Gonzalo Rojas.

19 "Nota de los editores", El Corno Emplumado, núm. 10, 1964, p. 5.

20 "Nota de los editores", El Corno Emplumado, núm. 10, 1964, p. 5. 
está nuestro destino, que debemos hacer nacer un hombre nuevo en nuestro interior. $^{21}$

En el mismo número, El Corno publicó una especie de declaración de principios del movimiento de Nueva Solidaridad:

Este Primer Encuentro deja como saldo valiosísimo el hecho de que todos los que nos habíamos comunicado por carta, nos hemos dado la mano, nos hemos conocido, hemos dialogado, y lo más importante. Hemos resuelto dejar de lado todos los aspectos formales y superficiales que aparentemente nos distanciaban, para unirnos en torno a nuestras coincidencias y hacer que tal diálogo sea permanente, a fin de que en futuros encuentros se consolide esta amistad y fraternidad constructivas. ${ }^{22}$

En esta declaración de Nueva Solidaridad, que no es casual que publique El Corno, salen a relucir importantes nociones que defendió la revista en torno a su idea de solidaridad intelectual. La que más destaca en primer lugar es la necesidad de una solidaridad intelectual que partiera de una toma de conciencia. La intención que los había unido en el movimiento y el encuentro formaba parte de una necesidad de superar la incomunicación entre los intelectuales del continente y las visiones nacionalistas de la cultura.

La revolución de El Corno y de esta red de solidaridad no era socialista o marxista, no tenía banderas ideológicas sino banderas de la responsabilidad humanas: "[...] Nueva Solidaridad no es un partido político captador del voto y la voluntad de mentalidades desorientadas, sino una alianza de seres responsables. Se es solidario por convicción propia y sin duda muchos cerrarán sus ojos a la evidencia. No se está proponiendo una medicina masiva, sino que dentro de la propia capacidad humana, se intenta contribuir a la paz, la comprensión y la justicia humanas". ${ }^{23}$

La declaración también dejó claro de que el movimiento Nueva Solidaridad no respondía a ninguna consigna, partido, o afiliación política. El único compromiso que expresaban lo miembros del movimiento era el logro de la

21 "Nota de los editores", El Corno Emplumado, núm. 10, 1964, p. 5.

22 "Primer encuentro americano de poetas. Movimiento NS (Nueva Solidaridad). Declaración de México", El Corno Emplumado, núm. 10, 1964, p. 112.

23 "Primer encuentro americano de poetas. Movimiento NS (Nueva Solidaridad). Declaración de México”, El Corno Emplumado, núm. 10, 1964, p. 113. 
paz a través del arte. A pesar de ello dejaron claro que no estaban ajenos a un contexto que los atravesaba:

Es importante decir que esta revolución es algo más que literaria: incluye la lucha de los negros estadounidenses por la igualdad de derechos, la lucha de pueblos sometidos a centenarias cadenas coloniales por su libertad, la lucha de todos los pacifistas del mundo por una justicia social y el desarme, los nuevos descubrimientos en el área de la sicología, y la lucha de marxistas, católicos, estudiantes y seres humanos de diverso origen y edad frente a una sociedad cuyas presiones son más y más mecánicas, y cuyas demandas más y más deshumanizantes. ${ }^{24}$

La libertad de creación del artista, aquella que sería tan debatida por otras redes intelectuales, ${ }^{25}$ fue otros de los signos más claros de El Corno y de la propuesta de Nueva Solidaridad. De esta forma remarcaban la creación literaria o artística sin ataduras ideológicas y la plena autonomía del autor para seleccionar las formas que considerara oportunas y trascedentes.

Los objetivos inmediatos que se empezaron a trazar tras el encuentro fueron la edición de la revista NS, de carácter continental, la cual sería editada por los puntos principales de esta red en el siguiente orden: Buenos Aires, México, Washington, Caracas y Managua. Una revista que por su carácter continental no radicaría en un solo país y haría mucho más efectiva la acción de las revistas ya existentes y la de sus miembros. La publicación nunca llegó a ver la luz pública pero indudablemente nos demuestra el alcance transnacional al que aspiraron los participantes de esta nueva solidaridad. Finalmente, la declaración terminaba con el aforismo "Paz a través del arte" y fue firmado por Eco Contemporáneo, El Corno, Pájaro Cascabel, El Rehilete, Outcry, Gonzalo Arando Alberto Hoysos en Colombia y El techo de la ballena de Venezuela.

Esta red, nombrada por la investigadora argentina Valeria Manzano como contracultural, no ha tenido mucha presencia en los estudios sobre redes intelectuales en los sesenta. Ellos se han centrado principalmente en la red intelectual que se nutrió alrededor de la revolución cubana con revistas

24 "Primer encuentro americano de poetas. Movimiento NS (Nueva Solidaridad). Declaración de México”, El Corno Emplumado, núm. 10, 1964, pp. 112.

${ }^{25}$ La revista Lunes de Revolución (1959-1961) promovió en Cuba un amplio debate sobre la libertad de creación y los límites del compromiso intelectual. Casa de las Américas, también desde Cuba, publicó varios trabajos al respecto. 
como Casa de las Américas, Marcha y Mundo Nuevo, las cuales fueron activas en torno a los debates sobre el intelectual y la revolución. Valdría la pena preguntarse por qué no se ha estudiado más. Una suposición pudiera ser que uno de los rasgos fundamentales de los sesenta fue la politización de la vida cultural, del mundo estudiantil, intelectual y artístico. A pesar de su activa participación pública, esta red de nueva solidaridad se mantuvo al margen de cierta politización de la vida cultural relacionada con términos como revolución cubana, izquierda, etc. Aunque en el caso de El Corno, progresivamente, y a partir del año 1966 iría mostrando cada vez más su apoyo al gobierno revolucionario, no se salió de su carril poético y contracultural, a la vez que abrió espacios para posturas críticas con el proyecto cubano.

La idea de revolución que circuló en esta red fue bien distinta a la que se irradiaba desde La Habana, como bien dejara claro El Corno:

Se habló, o quizás sólo se sugirió, la existencia de un hombre nuevo, se mencionó la nueva era, y se dijo que paralelamente a la reforma agraria era inaplazable una reforma agraria del espíritu. [sic] Transformar la sociedad? Por supuesto. Pero sobre la base de un profundo cambio en la naturaleza del hombre. Es decir, propusimos un método a la inversa de toda lógica: cambiándonos a nosotros mismos transformaremos el mundo en que vivimos. ${ }^{26}$

En los debates más conocidos de la época las alusiones a esta red de solidaridad y sus participantes o promotores fueron mínimas. Los únicos registros primarios son una antología de El techo de la ballena que preparó Ángel Rama y la cual no fue publicada hasta $1987,{ }^{27} \mathrm{y}$ algunas menciones aisladas en Casa de las Américas sobre El Corno Emplumado. Miguel Grinberg fue invitado a ser jurado del premio literario de Casa de las Américas en 1965, pero este es un episodio censurado de la historia de la literatura cubana, pues a ese mismo jurado fue invitado Allen Ginsberg, el cual terminó siendo expulsado del país por sus comentarios críticos a la figura de Fidel Castro y sus relaciones con un grupo de escritores underground, en su mayoría homosexuales y negros que se reunían alrededor de la editorial El Puente.

26 "Nota de los editores", El Corno Emplumado, núm. 10, 1964, p. 5.

27 Ángel Rama había seleccionado los escritos desde 1974, y al no encontrar un editor para el volumen, publicó solamente un texto sobre los balleneros (como comúnmente se les decía) que llamó "Prólogo". Finalmente, el proyecto no fructificaría y la antología no sería publicada hasta 1987, después de su muerte. 
Aunque Valeria Manzano ha adelantado un importante camino en historiar esa red contracultural de revistas y movimiento de solidaridad en los sesenta, aún queda mucho por hacer al respecto, la primacía la han tenido las revistas vinculadas a la red intelectual que se nutrió alrededor de Cuba. Los estudios sobre los sesenta se han concentrado en la red intelectual "hegemónica" que se articuló desde revistas vinculadas al proceso cubano como Casa de las Américas, Marcha y en las que entraron en polémicas con ellas, Mundo Nuevo o Libre. Este vacío historiográfico responde al protagonismo de estas últimas y a una herencia que concentra su mirada a los sesenta en torno al paradigma revolucionario que fomentó la revolución cubana.

\section{LA CORRESPONDENCIA SOLIDARIA}

La sección de correspondencia fue una de las más llamativas y polémicas de la revista. En ella se publicaron las misivas que escritores o artistas les enviaban al Corno y la de los lectores más frecuentes, lo cual permite acceder a la recepción de la revista y su impacto en la intelectualidad latinoamericana. La sección pretendía ser "un lugar donde los poetas podían expresar criterios divergentes y defender sus ideas sobre el arte y la sociedad. Esas ideas a veces eran discrepantes, y alentábamos esos desacuerdos planteados por uno u otro" (Randall, 2015, p. 105). Esta característica fue recurrente en la publicación que utilizó la sección como un medio de convertir a la revista en un espacio de diálogo intelectual. El hecho que publicaran cartas que pudieran considerarse privadas nos habla de la intencionalidad de la revista de mostrar los afectos que se generaron alrededor de la conformación de esta comunidad intelectual, sus diálogos, discusiones e incluso sus formas de vida.

Las cartas se convirtieron en una suerte de espacio íntimo de la revista y de sus lectores. Al hacer público lo que puede pensarse como privado se hizo latente la red de solidaridad intelectual que tejió El Corno. En misiva de Ernesto Cardenal a Sergio Mondragón, el poeta nicaragüense le comentaba:

Te diré: ustedes están creando la verdadera Unión Panamericana. La Unión Panamericana es la de los poetas, no la de esos que se sientan en los banquetes y "devoran a mi pueblo como si fuera pan" como dice el Salmo. Los poetas son los que se entienden, a pesar de las barreras del idioma, porque ellos son los que tienen los órganos de comunicación, son la voz de la tribu. Si los poetas 
no realizan el Panamericanismo nadie más lo hará. Y lo están haciendo. Y por primera vez en la historia se comenzarán a entender el pueblo norteamericano y el hispanoamericano, en un verdadero entendimiento de pueblos, porque se entienden sus poetas. ${ }^{28}$

A través de este espacio también podemos rastrear las discusiones de esta red y cómo sus vínculos se estrechaban cada vez más. La correspondencia fue fundamental para articular este movimiento de solidaridad y es posiblemente la fuente más tangible de cómo se articuló esta red y qué objetivos persiguió. En ella encontramos las cartas de Grinberg a El Corno sobre los preparativos del I Encuentro de Poetas, o los comentarios de los nadaístas sobre el movimiento de Nueva Solidaridad. A la vez permite rastrear los recorridos individuales de las figuras que conformaron la red.

El uso de la correspondencia para trazar esos lazos de fraternidad fue fundamental en El Corno. Ella fue un medio de vínculo y diálogo entre las diferentes latitudes de esta red, a la vez la solidaridad se expresó muchas veces en las cartas que se publicaban. Además de las cartas de los lectores, en las que narraban no sólo sus impresiones sobre El Corno, sino también sus últimas hazañas: la lectura de un ejemplar o la participación en una manifestación, se publicaba la correspondencia entre los propios colaboradores en la que se expresaban de forma muy personal. Fueron habituales las cartas entre Mondragón, Arango, Grinberg y también del ballenero Edmundo Aray, el poeta ecuatoriano Ulises Estrella, y los más consagrados Ernesto Cardenal y Roque Dalton.

Después de El Primer Encuentro de Poetas, con el apoyo de Margaret Randall, Miguel Grinberg se dirigió a Estados Unidos para entablar contacto con miembros de la Generación Beat y en su primera misiva publicada en El Corno le comentaba:

Los revolucionarios políticos nos seguirán acometiendo, pues de alguna manera se dan cuenta que quedaron rezagados. Nuestra firmeza, nuestra unión y nuestras realizaciones futuras serán el argumento que les haga no temer la reforma agraria del espíritu de que hablamos. Y debemos seguir creando,

${ }^{28}$ Ernesto Cardenal, "Carta”, El Corno Emplumado, núm. 5, 1962, p. 146. 
uniendo la acción a la palabra, tanto en el hogar como en la calle, tanto en la meditación solitaria como en el diálogo con los demás. ${ }^{29}$

La recepción del viaje de Grinberg a Estados Unidos no se haría esperar y en 1964 Thomas Merton le escribía a Margaret y Sergio:

It was great to have Miguel [Grinberg] come through Kentucky. Ron brought him down in cataracts of rain and it was so dark you could hardly see anything but we took some dark pictures and spoke of the Encuentro and the New Man. The idea of the New Solidarity is impressive and real. There can be no renewal of life and intelligence until poets and artists have restored... freedom and unpredictability wich have been destroyed by the politicians. ${ }^{30}$

\section{A MODO DE CIERRE}

A partir de 1964 la red intelectual alrededor de El Corno se fue resquebrajando y el proyecto de Nueva Solidaridad se disgregó de acuerdo con los distintos caminos intelectuales de sus miembros. En una carta publicada en julio de 1965, Grinberg manifestaba su inconformidad con los reclamos políticos que se percibían en los discursos de los escritores de la época, a la vez que manifestaba cierta decepción de su viaje por Estados Unidos y disconformidad con muchos de los preceptos que se venían manejando en el círculo intelectual a su alrededor:

La nueva solidaridad nunca deberá ser una bandera. Deberá ser un sentimiento silencioso. Discutir y negar al prójimo es convertirse en embajador de la muerte. [...] Hay mucho fanatismo y sordera en todas partes. Sí, una vez LeRoi Jones me escribió que el Corno necesitaba "a stronger editorial hand". Sí, se lo escribí a Ernesto. Sí, no te lo escribí a vos. Tanto el Corno como el Eco han publicado cosas malas, no es novedad. No tengo nada que criticarte. Es muy fácil opinar cuando el que se rompe el lomo sacando una revista es otro. Le comenté eso a Cardenal antes de conocer personalmente a un montón de gente.

${ }^{29}$ Miguel Grinberg, "Carta”, El Corno Emplumado, núm. 10, 1964, p. 118.

30 Thomas Merton, "Carta”, El Corno Emplumado, núm. 11, 1964, p. 154. 
Pero después de 100 días con los poetas de Nueva York, después de estar con los poetas nicaragüenses y de mucho más [...] vengo dándome cuenta que en muchos casos la poesía es un buen pretexto para encubrir la pereza espiritual y la pobreza psíquica. Muchos que parecían ser maravillosos humanistas por carta, personalmente resultaron vacíos, resentidos y destructivos. El verdadero poeta lo es sin necesidad de pregonarlo. El verdadero revolucionario lo es sin publicitario. ${ }^{31}$

No obstante, el diálogo entre una parte de esta red continuaría, al igual que Grinberg, Gonzalo Arango desde Colombia se mantuvo presente en la sección de "Cartas" y a la altura de 1966 les decía:

A mí, personalmente, el Corno me ha hecho sentir más americano, ligado a una nueva dimensión espiritual de poeta y de hombre, pues la fraternidad que no ha logrado la ozA entre nuestros pueblos, la he sentido palpitar en la insumisión y la belleza de esas oleadas de poesía que nos trae el Corno cada vez, y que nos pone ante el espejo de nuestra realidad neta, brutal, insobornable, conquistando nuestro destino en el arte y en la vida, como poetas maravillosos y como maravillosos pueblos. ${ }^{32}$

En enero de 1965, en su nota editorial, la revista festejaba su alcance y pronunciaba la dicha de lo logrado, para El Corno, la verdadera victoria era haber establecido una comunicación tan variada y prolífera entre la poesía de América:

[...] la labor que en un principio parecía imposible para una pequeña revista literaria independiente, ha sido felizmente realizada: tres años ininterrumpidos de trabajo, 13 números de El Corno Emplumado puntualmente aparecidos y convencionalmente distribuidos en los distintos países del continente, y lo que es más interesante: la comunicación que este hecho ha propiciado entre los diversos poetas y movimientos poéticos de América, lo que solo es una pequeña parte del fenómeno humano más importante de los últimos 2,000 años: la revolución espiritual de la conciencia contemporánea. ${ }^{33}$

\footnotetext{
${ }^{31}$ Miguel Grinberg, “Carta”, El Corno Emplumado, núm. 15, 1965, pp. 147-148.

32 Gonzalo Arango, "Carta”, El Corno Emplumado, núm. 18, 1966, p. 232.

33 “Nota de los editores”, El Corno Emplumado, núm. 13, 1965, p. 5.
} 
Aunque el proyecto de solidaridad de El Corno estuvo mayormente ligado a la red intelectual que se gestó en torno a Nueva Solidaridad, iría mutando a otros presupuestos en consonancia con la evolución misma de la idea de solidaridad en la época. Recordemos que a partir de 1965 se comienzan a gestar con más fuerzas encuentros como la Conferencia Tricontinental o la Organización de Solidaridad de los Pueblos de África, Asia y América Latina que reforzaron una idea de solidaridad vinculada a la revolución cubana. En próximas notas editoriales, la voz solidaria de El Corno giraría a condenar la expulsión de Cuba de la oza o la guerra en Vietnam. Los nombres del Che o Regis Debray comenzarían a ser los nuevos paradigmas intelectuales, y el número 18, en abril de 1966, estaría dedicado al asesinato del cura guerrillero Camilo Torres.

Uno de los factores que influyó en esta resignificación de la noción de solidaridad fue el creciente apoyo de El Corno a la revolución cubana. Para mediados de la década cada vez se hacía más difícil construir un espacio intelectual que no estuviera plagado por el tema "Cuba". Uno de los más emblemáticos posicionamientos al respecto fue la publicación de un número entero dedicado a la poesía cubana. Aunque era habitual que El Corno ofreciera números consagrados a la literatura de varias naciones, el dedicado a Cuba, desde su portada, ${ }^{34}$ su nota editorial o la configuración del índice, demostraba una clara toma de postura a favor del proceso revolucionario. En la nota editorial expresaban: "el corno emplumado ofrece desde México este número como su homenaje al 26 de julio, y en un modesto intento de romper el bloqueo cultural que, paralelo al económico, han impuesto a Cuba los gobiernos del continente americano, como medida desesperada ante su propia impotencia, [...] vamos a dejar a la poesía cubana hablar por sí misma". ${ }^{35}$

El ejemplar incluyó una amplia selección de poetas cubanos, la crónica "Cuba: impressions eight years from triumph", de Margaret Randall, una declaración del consejo de colaboración de la revista Casa de las Américas condenando el militarismo de la oza y acciones de la Alianza para el Progreso en América Latina y las reseñas de los libros ¿Revolución en la revolución?, de

${ }^{34}$ La portada del número estuvo a cargo de Felipe Ehrenberg y con llamativos colores mostraba una bandera cubana junto a unos hombres barbudos en clara alusión metafórica a "lo revolucionario".

35 “Nota de los editores", El Corno Emplumado, núm. 23, 1967, p. 5. 
Regis Debray, y Paradiso, de José Lezama Lima. Sobre este, Margaret Randall recuerda:

Este número de $\mathrm{El}$ Corno tiene una historia interesante. Habíamos publicado escritores cubanos anteriormente, y también había vendido 500 suscripciones a la revista a la Unión Panamericana, el brazo cultural de la Organización de Estados Americanos (OEA). La Unión se opuso a que ofreciéramos un foro a los escritores cubanos, y amenazó con cancelar sus suscripciones si continuamos publicándolos. No nos comprarían y cancelaron sus suscripciones. La revista continuó su camino independiente (Mondragón y Randall, 2020).

El número se conformó tras la visita de Randall a La Habana en enero de 1967 para asistir a una reunión de poetas y críticos literarios en homenaje al aniversario del nacimiento de Rubén Darío. El ejemplar aparecería en julio en honor a la fecha revolucionaria cubana del 26 de julio. La poeta regresaría a Cuba en 1968 para el Congreso Cultural de La Habana y en 1969 volvería tras el cierre de El Corno llegando a vivir casi toda la década del setenta en la isla.

El proyecto de solidaridad de la revista, aunque más cohesionado en sus primeros cuatro años, intentó unificar la revolución espiritual con la comunicación y la fraternidad entre escritores y artistas. Como "verdaderos embajadores de solidaridad continental" se trazaron una misión que superó las expectativas de sus mismos propósitos y logró nuclear a una vasta red intelectual que terminó imponiendo algunas de las características más importantes del universo intelectual latinoamericano de los sesenta del siglo xx. En 1969, El Corno Emplumado llegó a su fin, principalmente por la censura política. Tras haber apoyado el movimiento estudiantil del 68 perdió casi todo su financiamiento y la mayoría de las imprentas se negaron a imprimir la revista. Los tiempos cambiaban y los sucesos del año anterior impondrían nuevas dinámicas al campo intelectual y sus redes.

\section{LISTA DE REFERENCIAS}

Beigel, F. (2003). Las revistas culturales como documentos de la historia latinoamericana. Utopía y Praxis Latinoamericana, 8(20), 105-115. Recuperado de https://www. redalyc.org/pdf/279/27902007.pdf 
Crespo, R. (coord.) (2010). Revistas en América Latina: proyectos literarios, políticos y culturales. México: CIALC-UnAm.

Devés-Valdés, E. (2007). Redes intelectuales en América Latina. Hacia la constitución de una comunidad intelectual. Santiago de Chile: Instituto de Estudios AvanzadosUniversidad Santiago de Chile.

Gilman, C. (2003). Entre la pluma y el fusil. Debates y dilemas del escritor revolucionario en América Latina. Buenos Aires: Siglo XXI (Colección Metamorfosis).

León, I. de (2017). Redes intelectuales en América Latina: una lectura desde los márgenes. En L. Weinberg (coord.), El ensayo en diálogo. México: CiALC-UNAM.

Manzano, V. (2017) Fraternalmente americanos: el Movimiento Nueva Solidaridad y la emergencia de una contracultura en la década de 1960. Iberoamericana, XVII(66), 115-138.

Marchesi, A. (2019) Hacer la revolución. Guerrillas latinoamericanas, de los años sesenta a la caída del Muro. Buenos Aires: Siglo XXI Editores.

Mondragón, S y Randall, M. (2020). El Corno Emplumado 23. Open Door Archive, Recuperado de https://opendoor.northwestern.edu/archive/items/show/58

Pereira, A., Albarrán, C., Rosado, J. A. y Tornero, A. (2018). El Corno Emplumado. elem.mx. Enciclopedia de la Literatura en México. Recuperado de http://www.elem. $\mathrm{mx} /$ institucion/datos/179424

Pirker, K. (diciembre de 2017-mayo de 2018). Activismo transnacional y solidaridad, de Cuba a Centroamérica. Revista de la Red de Intercátedras de Historia de América Latina Contemporánea, 4(7), 120-138.

Pita, A. (comp.). (2016). Redes intelectuales transnacionales en América Latina durante la entreguerra. México: Universidad de Colima.

Randall, M. (2015). Recordando El Corno Emplumado. Casa de las Américas, 280, 100-118.

Santos, J. (2002). Intelectuales y prensa en el siglo xx. En C. Almuiña y E. Sotillos (coords.), Del periódico a la sociedad de la información (pp. 197-218). Madrid: Sociedad Estatal Nuevo Milenio.

Silva, G. (2017). Texto, contexto e índices de El Corno Emplumado (1962-1969). (Tesis de maestría), El Colegio de San Luis, A.C., San Luis de Potosí, México. Recuperado de https://biblio.colsan.edu.mx/tesis/SilvaIbarguenGabriela.pdf

Thompson, J. (2002). Ideología y cultura moderna. Teoría Crítica social en la era de la comunicación de masas. México: Universidad Autónoma Metropolitana. 